\title{
Secure Communications via Stochastic Constrained Uncertain Hyperchaotic System
}

\author{
Mohamed Fahim Hassan* \\ Electrical Engineering Department, College of Engineering, Kuwait University. P.O.Box: 5969 Safat, 13060 \\ Kuwait. \\ * Corresponding author. Tel.: +965-2498-7634; email: m.f.hassan47@gmail.com \\ Manuscript submitted October 8, 2016; accepted December 18, 2016. \\ doi: 10.17706/ijcee.2016.8.6.319-329
}

\begin{abstract}
In this paper, a secure communication scheme is proposed in which the concept of carrier encryption is used. At the transmitter end, the unified chaotic system and the uncertain hyperchaotic Chen system are coupled, constrained and used as a new hyperchaotic system. The outputs of the system are encrypted using a set of pre-defined encryption rules. The encrypted outputs are transmitted to the receiving end through a noisy public communication channel. At the receiving end, the Iterative Decomposed Uncertain Constrained Extended Kalman Filter (IDUCEKF) is used to reconstruct the carrier signals and hence retrieve the transmitted data. A simulated case study is presented where the proposed secure communication scheme is applied to transmit discrete images. The quality of the proposed communications scheme is measured by the bit-error rate (BER) and the associated correlation coefficients.
\end{abstract}

Key words: Extended Kalman filter, state estimation, secure communications, chaos synchronization.

\section{Introduction}

Security in Chaos-based communications has recently gained an increased interest from researchers [1], [2]. Most of the chaos-based communications schemes are based on chaos synchronization between transmitter and receiver [3]-[11]. In that regard, the techniques used for chaos synchronization can be classified into control techniques [3]-[6], estimation techniques such as extended Kalman filter (EKF) [2], [7]-[9], and artificial intelligent approaches [10] among others. In these approaches, masking the contents of a message using chaotic signals have been achieved using different methods [7], [10]. However, it has been shown that most of these techniques are not secure since it is possible to extract the encoded message signal from the transmitted chaotic signal by using different unmasking techniques [12], [13]. So, to overcome this problem, different approaches have been developed to design cryptosystems based on chaos [2], [11]. In these approaches, conventional cryptographic method and synchronization of chaotic systems are combined in order to enhance the security level of transmitted chaotic signal.

In this paper, a secure chaos-based communications system is proposed where the chaotic oscillator is also encrypted before being used to mask the signal. The encryption of the oscillator is accomplished by: a) coupling two different chaotic oscillators, b) introducing uncertainty to the system's parameters, and c) applying a set of equality and/or inequality constraints on the unmodulated chaotic signals. Such a combination leads to a chaotic signal which is very difficult to synchronize without having full information about the encryption process. The outputs of the system including that modulated by the encrypted data 
are encrypted before being transmitted to the receiving end through a public noisy channel. At the receiving end, the decrypted received outputs are used to reconstruct the hyperchaotic signals using the newly developed Iterative Decomposed Uncertain Constrained Extended Kalman Filter (IDUCEKF), and hence extract the data signal. The quality of the proposed secure communications scheme is assessed through the transmission of two coloured images.

The rest of the paper is organized as follows. The proposed secure communication scheme and the IDUCEKF are presented in sections 2 and 3, respectively. In section 4, simulation results are demonstrated to show the effectiveness of the proposed secure communication scheme. Finally, the paper is concluded in section 5 .

\section{The Proposed Secure Communication Scheme}

In this section, we present a brief description of the proposed communication scheme in which the hyperchaotic Chen system and the unified chaotic system are coupled to form a new hyperchaotic system. The discrete model of the coupled uncertain hyperchaotic system is given by: [11]

$$
\begin{aligned}
& x_{u 1 k+1}=x_{u 1 k}+\Delta T(25 \alpha+10)\left(x_{u 2 k}-x_{u 1 k}\right)+w_{u 1 k} \\
& x_{u 2 k+1}=x_{u 2 k}+\Delta T(28-35 \alpha) x_{h 1 k}-\Delta T x_{h 1 k} x_{u 3 k}+\Delta T(29 \alpha-1) x_{u 2 k}+w_{u 2 k} \\
& x_{u 3 k+1}=x_{u 3 k}+\Delta T x_{h 1 k} x_{u 2 k}-((8+\alpha) / 3) \Delta T x_{u 3 k}+w_{u 3 k} \\
& x_{h 1 k+1}=(1-\Delta T a) x_{h 1 k}+\Delta T a x_{h 2 k}+\Delta T x_{h 4 k}+w_{h 1 k} \\
& x_{h 2 k+1}=\Delta T d x_{h 1 k}+(1+\Delta T c) x_{h 2 k}-\Delta T x_{h 1 k} x_{h 3 k}+w_{h 2 k} \\
& x_{h 3 k+1}=\Delta T x_{h 1 k} x_{h 2 k}+(1-\Delta T b) x_{h 3 k}+w_{h 3 k} \\
& x_{h 4 k+1}=\Delta T x_{h 2 k} x_{h 3 k}+(1+\Delta T r) x_{h 4 k}+w_{h 4 k}
\end{aligned}
$$

The outputs of the coupled hyperchaotic system are:

$$
\begin{aligned}
& y_{u 1 k+1}=0.1 x_{u 1 k+1}, y_{u 2 k+1}=0.1 x_{u 2 k+1}, \\
& y_{h 1 k+1}=0.1 x_{h 1 k+1}, y_{h 2 k+1}=0.1 x_{h 2 k+1}
\end{aligned}
$$

The parameter $\alpha$ is adaptive, and its formula is given by:

$$
\alpha=\left|x_{h 1 k}\right| /\left(20\left(\left|x_{h 1 k}\right|+1\right)\right)
$$

The generated chaotic signals resulting from the hyperchaotic system (1) are subject to the following set of constraints:

$$
\underline{\eta}_{k+1} \leq \Xi_{k+1}\left(x_{u k+1}, x_{h k+1}\right) \leq \bar{\eta}_{k+1}
$$

where $\boldsymbol{x}_{u k}, \boldsymbol{x}_{h k}, \boldsymbol{y}_{u k+1}, \boldsymbol{y}_{h k+1}$ are the state and output vectors of the unified Chaotic system and the hyperchaotic Chen system, respectively, $\boldsymbol{w}_{u k}, \boldsymbol{w}_{h k}$ are zero mean uncorrelated white Gaussian input noise vectors with covariance matrices $\boldsymbol{Q}_{u}=\boldsymbol{E}\left\{\boldsymbol{w}_{u} \boldsymbol{w}_{u}^{T}\right\}, \boldsymbol{Q}_{h}=\boldsymbol{E}\left\{\boldsymbol{w}_{h} \boldsymbol{w}_{h}^{T}\right\}$, respectively, $\alpha$ is the unified chaotic system parameter, $a, b, c, d$ and $r$ are the parameters of the Chen system assumed to be uncertain with means and variances to be specified in section $4, \boldsymbol{\Xi}_{k+1}: \boldsymbol{R}^{7} \rightarrow \boldsymbol{R}^{j}$ is the constrained vector nonlinear function; $j$ is the number of constraints; $\underline{\eta}_{k+1}, \bar{\eta}_{k+1}$ are, respectively, the vectors of lower and upper bounds of $\Xi_{k+1}$ element by element which can be time dependent, and $\Delta T$ is the sampling time period.

The unified chaotic system is chaotic for any value of $\alpha \in[0,1]$. For the hyperchaotic Chen system, if the 
mean values of the parameters are equal to $\bar{a}=35, \bar{b}=3, \bar{c}=12, \bar{d}=7$ and $0.085 \leq \bar{r}<0.798$, the system is hyperchaotic.

One of the outputs of the hyperchaotic system, say $y_{h 2 k+1}$, is modulated by the encrypted data signal $S T(k)$ which leads to the modulated output signal $y_{h 2 k+1}^{m}$. The modulated and the unmodulated outputs of the hyperchaotic systems are encrypted using an appropriate encryption rule to be chosen by the designer. The encrypted outputs are transmitted to the receiving end through a noisy public transmission channel. At the receiving end, the received outputs are decrypted using the decryption rule. The IDUCEKF is used as an engine to reconstruct and synchronize the states of the constrained uncertain coupled hyperchaotic system given by (1) while using the decrypted un-modulated outputs. The received decrypted modulated output denoted by $y_{h 2 k+1}^{d m}$ and the estimated output $\hat{y}_{h 2 k+1 k+1}$ are used to retrieve the received encrypted data signal $S T_{n}(k)$. The decryption rule of the data signal is employed to get the recovered information signal $S_{n}(k)$. Finally, we filter the recovered information signal by using a threshold detector to retrieve the original signal $S(k)$.

\section{The Iterative Decomposed Uncertain Constrained EKF}

The IDUCEKF is a two phase algorithm. In the first phase, the states of the uncertain discrete-time nonlinear system are estimated using the iterative decomposed uncertain EKF (IDUEKF). If a subset of the constraints or the entire set is violated, we go to the second phase of the IDUCEKF in which the decomposed nonlinear update of the estimator is used.

\subsection{The Dynamics of the Iterative Decomposed Uncertain EKF}

Consider the following nonlinear discrete-time dynamical system in which the state vector is decomposed, either physically or mathematically or both, into $\mathrm{N}$ subsystems and the output measurement vector into M sub-vectors. Then we get:

$$
\begin{gathered}
\boldsymbol{x}_{i, k}=\boldsymbol{f}_{i, k}\left(\boldsymbol{x}_{k}, \boldsymbol{b}_{\boldsymbol{k}}\right)+\boldsymbol{w}_{i, k} i \in\{1,2, \ldots, N\} \\
\boldsymbol{y}_{r, k+1}=\boldsymbol{h}_{r, k+1}\left(\boldsymbol{x}_{k+1}, \boldsymbol{c}_{k+1}\right)+\boldsymbol{v}_{r, k+1} \quad r \in\{1,2, \ldots, M\}
\end{gathered}
$$

where $\boldsymbol{x}_{i, k}=\boldsymbol{x}_{i, k}(\boldsymbol{k}) \in \boldsymbol{R}^{\boldsymbol{n}_{i}}$ is the state vector of the $i^{\text {th }}$ subsystem; $n=\sum_{i=1}^{N} n_{i} ; \boldsymbol{y}_{r, k+1} \in \boldsymbol{R}^{\boldsymbol{m}_{r}}$ is the observation model of the $r^{\text {th }}$ measurement subsystem, $m=\sum_{r=1}^{M} m_{r}$. The vectors $\boldsymbol{w}_{\boldsymbol{i}, \boldsymbol{k}} \in \boldsymbol{R}^{\boldsymbol{n}_{\boldsymbol{i}}}$ and $v_{r, k} \in \boldsymbol{R}^{\boldsymbol{m}_{r}}$ are the input and output noise vectors with covariance matrices $\boldsymbol{E}\left\{\boldsymbol{w}_{i, k} \boldsymbol{w}_{i, k}^{T}\right\}=\boldsymbol{Q}_{i i, k}$ and $\boldsymbol{E}\left\{v_{r, k} v_{r, k}^{T}\right\}=\boldsymbol{R}_{r r, k}$, respectively. The vectors functions $\boldsymbol{f}_{i, k}$ and $\boldsymbol{h}_{r, k}$ are such that: $\boldsymbol{f}_{i, k}\left(\boldsymbol{x}_{k}\right): \boldsymbol{R}^{n} \rightarrow \boldsymbol{R}^{n_{i}}$ and $\boldsymbol{h}_{r, k}\left(\boldsymbol{x}_{\boldsymbol{k}}\right): \boldsymbol{R}^{n} \rightarrow \boldsymbol{R}^{\boldsymbol{m}_{r}} ; \boldsymbol{b}_{\boldsymbol{k}} \in \boldsymbol{R}^{\gamma}$ is the uncertain parameter vector of the system model assumed white Gaussian with mean $\bar{b}$ equals to the nominal values of the model parameters, and a covariance matrix $\Gamma_{\boldsymbol{k}}=\boldsymbol{E}\left\{\left(\boldsymbol{b}_{\boldsymbol{k}}-\overline{\boldsymbol{b}}\right)\left(\boldsymbol{b}_{\boldsymbol{k}}-\overline{\boldsymbol{b}}\right)^{T}\right\} ;$ and $\boldsymbol{c}_{\boldsymbol{k}} \in \boldsymbol{R}^{u}$ is the uncertain parameter vector of the output model assumed white Gaussian with mean $\bar{c}$, equals to the nominal values of the output model parameters and a covariance matrix $\boldsymbol{U}_{k}=\boldsymbol{E}\left\{\left(\boldsymbol{c}_{\boldsymbol{k}}-\overline{\boldsymbol{c}}\right)\left(\boldsymbol{c}_{\boldsymbol{k}}-\overline{\boldsymbol{c}}\right)^{T}\right\}$. Moreover, it is assumed that the initial condition $\boldsymbol{x}_{\boldsymbol{o}}$ is a Gaussian random vector with zero mean and covariance $\boldsymbol{E}\left\{\boldsymbol{x}_{\boldsymbol{o}} \boldsymbol{x}_{\boldsymbol{o}}^{T}\right\}=\boldsymbol{P}(0)$. Finally, $k \in\{0,1,2, \ldots\}$ denotes 
the discrete time instant.

In the above model, it is assumed that $\boldsymbol{x}_{o}, \boldsymbol{b}_{\boldsymbol{k}}, \boldsymbol{c}_{\boldsymbol{k}+1}, \boldsymbol{w}_{k}, v_{k+1}$ are all independent. Moreover, it is assumed that the model has the following properties:

$$
\begin{array}{ll}
\boldsymbol{E}\left\{\boldsymbol{x}_{\boldsymbol{k}} \boldsymbol{w}_{j}^{T}\right\}=0, \forall \boldsymbol{k} \leq \boldsymbol{j} ; & \boldsymbol{E}\left\{\boldsymbol{x}_{\boldsymbol{k}} \boldsymbol{v}_{j}^{T}\right\}=0, \forall \boldsymbol{k}, \boldsymbol{j} ; \boldsymbol{E}\left\{\boldsymbol{x}_{\boldsymbol{k}} \boldsymbol{c}_{j}^{T}\right\}=0, \forall \boldsymbol{k}, \boldsymbol{j} ; \\
\boldsymbol{E}\left\{\boldsymbol{y}_{\boldsymbol{k}} \boldsymbol{v}_{j}^{T}\right\}=0, \forall \boldsymbol{k}<\boldsymbol{j} ; & \boldsymbol{E}\left\{\boldsymbol{x}_{\boldsymbol{k}} \boldsymbol{b}_{j}^{T}\right\}=0, \forall \boldsymbol{k} \leq \boldsymbol{j} ; \boldsymbol{E}\left\{\boldsymbol{y}_{k} \boldsymbol{c}_{j}^{T}\right\}=0, \forall \boldsymbol{k}<\boldsymbol{j}
\end{array}
$$

Assume that the last filtered state estimate and its associated covariance matrix are given by $\hat{\boldsymbol{x}}(\boldsymbol{k} \mid \boldsymbol{k})$ and $\boldsymbol{P}(\boldsymbol{k} \mid \boldsymbol{k})$, respectively. Moreover, we assume that the conditional probability density function $\boldsymbol{p}\left(\boldsymbol{x}_{\boldsymbol{k}} \mid \boldsymbol{Y}^{k}\right)$ is approximated by a Gaussian distribution with conditional mean $\hat{\boldsymbol{x}}(\boldsymbol{k} \mid \boldsymbol{k})$ and with covariance matrix $\boldsymbol{P}(\boldsymbol{k} \mid \boldsymbol{k})$.

The following lemma gives the dynamics of the IDUEKF.

\section{Lemma 3.1}

1) The predicted estimate of the states of the nonlinear discrete-time dynamical subsystems (5) are given by:

$$
\left.\hat{\boldsymbol{x}}_{i, k+1 \mid k} \cong f_{i, k}\left(\hat{\boldsymbol{x}}_{\boldsymbol{k} \mid \boldsymbol{k}}, \overline{\boldsymbol{b}}\right) \quad \text { (for } i \in\{1,2, \ldots, N\}\right)
$$

2-a) The covariance matrices of the prediction error of the $i^{\text {th }}$ subsystems are given by:

$$
\boldsymbol{P}_{\tilde{x}_{i, k+1 \mid k} \tilde{x}_{i, k+1 \mid k}} \cong \hat{F}_{i, k}^{\prime} P_{k \mid k} \hat{F}_{i, k}^{\prime T}+\hat{E}_{i, k}^{\prime} \Gamma_{k} \hat{E}_{i, k}^{\prime T}+Q_{i i, k}
$$

where:

$$
\hat{\boldsymbol{F}}_{i, k}^{\prime}=\left[\left.\frac{\partial \boldsymbol{f}_{i, k}^{T}}{\partial \boldsymbol{x}_{k}}\right|_{\substack{x_{k}=\hat{x}_{k \mid k} \\ b_{k}=\bar{b}}}\right]^{T} \in \boldsymbol{R}^{n_{i} \times n} \text { and } \hat{\boldsymbol{E}}_{i, k}^{\prime}=\left[\left.\frac{\partial \boldsymbol{f}_{i, k}^{T}}{\partial \boldsymbol{b}_{k}}\right|_{\substack{x_{k}=\hat{x}_{k \mid k} \\ b_{k}=\bar{b}}}\right]^{T} \in \boldsymbol{R}^{n_{i} \times \gamma}
$$

2-b) The cross covariance matrices of the prediction error of the $i^{\text {th }}$ and the $j^{\text {th }}$ subsystems (for $j \in$ $\{\mathrm{i}+1,2, . ., \mathrm{N}\})$ are given by:

$$
\boldsymbol{P}_{\tilde{x}_{i, k+1 k} \tilde{x}_{j, k+1 \mid k}} \cong \hat{F}_{i, k}^{\prime} P_{k \mid k} \hat{F}_{j, k}^{\prime T}+\hat{E}_{i, k}^{\prime} \Gamma_{k} \hat{E}_{j, k}^{\prime T}
$$

where:

$$
\begin{gathered}
\hat{\boldsymbol{F}}_{\mathrm{j}, k}^{\prime}=\left[\left.\frac{\partial \boldsymbol{f}_{\mathrm{j}, k}^{T}}{\partial \boldsymbol{x}_{\boldsymbol{k}}}\right|_{\substack{x_{k}=\hat{\boldsymbol{x}}_{k \mid k} \\
b_{k}=\bar{b}}}\right]^{T} \in \boldsymbol{R}^{n_{j} \times n} \text { and } \hat{\boldsymbol{E}}_{\mathrm{j}, k}^{\prime}=\left[\left.\frac{\partial \boldsymbol{f}_{\mathrm{j}, k}^{T}}{\partial \boldsymbol{b}_{\boldsymbol{k}}}\right|_{\substack{x_{k}=\hat{\boldsymbol{k}}_{k \mid k} \\
b_{k}=\bar{b}}}\right]^{T} \in \boldsymbol{R}^{n_{j} \times \gamma} \\
\hat{\boldsymbol{x}}_{i, k+1 \mid k+1}^{0}=\hat{\boldsymbol{x}}_{i, k+1 \mid k}
\end{gathered}
$$

For the sub-vector of the measurement $y_{r, k+1}$ where $r \in\{1,2, \ldots . \mathrm{M}\}$,

3) The filtered estimates of the states of the nonlinear discrete-time dynamical subsystems are such that: 


$$
\begin{aligned}
& \hat{\boldsymbol{x}}_{i, k+1 \mid k+1}^{r} \cong \hat{\boldsymbol{x}}_{i, k+1 \mid k+1}^{r-1}+\boldsymbol{K}_{i, k+1}^{r} \tilde{\boldsymbol{y}}_{r, k+1 \mid k+1}^{r-1} \text { for } r \in\{1,2, \ldots . M\}
\end{aligned}
$$

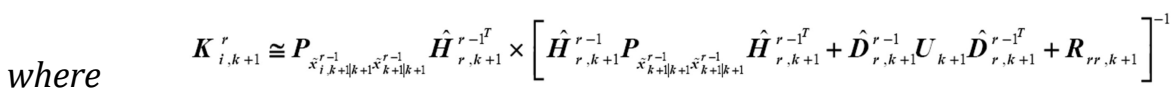

$$
\begin{aligned}
& \tilde{\boldsymbol{y}}_{r, k+1 \mid k+1}^{r-1} \cong \boldsymbol{y}_{r, k+1}-\hat{\boldsymbol{h}}_{r, k+1}\left(\hat{\boldsymbol{x}}_{\boldsymbol{k}+1 \mid k+1}^{r-1}, \overline{\boldsymbol{c}}\right) \\
& \hat{\boldsymbol{H}}_{\mathrm{r}, \boldsymbol{k}+1}^{r-1}=\left[\left.\frac{\partial \boldsymbol{h}_{\mathrm{r}, \boldsymbol{k}+1}^{T}}{\partial \boldsymbol{x}_{\boldsymbol{k}+1}}\right|_{\substack{x_{k+1}=\hat{x}_{k+1 \mid k+1}^{r-1} \\
\boldsymbol{c}_{k+1} \bar{c}}}\right]^{T} \in \boldsymbol{R}^{\boldsymbol{m}_{r} \times n} \text { and } \quad \hat{\boldsymbol{D}}_{\mathrm{r}, \boldsymbol{k}+1}^{r-1}=\left[\left.\frac{\partial \boldsymbol{h}_{\mathrm{r}, \boldsymbol{k}+1}^{T}}{\partial \boldsymbol{c}_{k+1}}\right|_{\substack{x_{k+1=}=\hat{x}_{k+1 \mid k+1}^{r-1} \\
\boldsymbol{c}_{k+1}=\bar{c}}}\right]^{T} \in \boldsymbol{R}^{\boldsymbol{m}_{r} \times u}
\end{aligned}
$$

4-a) The covariance matrices of the filtered error of the $i^{\text {th }}$ subsystems (for $\left.i \in\{1,2, . ., \mathrm{N}\}\right)$ are given by:

$$
\boldsymbol{P}_{\tilde{x}_{i, k+k \mid k+1}^{r}, \tilde{x}_{i, k+1 \mid k+1}^{r}} \cong \boldsymbol{P}_{\hat{x}_{i, k+1 \mid k+1}^{r-1}, \tilde{x}_{i, k+1 \mid k+1}^{r-1}}-\boldsymbol{K}_{i, k+1}^{r} \hat{\boldsymbol{H}}_{r, k+1}^{r-1} \boldsymbol{P}_{\tilde{x}_{k+1 \mid k+1}^{r-1}, \hat{x}_{i, k+1 \mid k+1}^{r-1}}
$$

4-b) The cross covariance matrices of the filtered error of the $i j^{\text {th }}$ subsystems (for $j \in\{\mathrm{i}+1,2, . ., \mathrm{N}\}$ ) are given by:

$$
\boldsymbol{P}_{\hat{x}_{i, k+1 \mid k+1}^{r}+\tilde{x}_{j, k+1 \mid k+1}^{r}} \cong \boldsymbol{P}_{\hat{x}_{i, k+1 \mid k+1}^{r-1}+\tilde{x}_{j, k+1 \mid k+1}^{r-1}}-\boldsymbol{K}_{i, k+1}^{r} \hat{\boldsymbol{H}}_{\mathrm{r}, \boldsymbol{k}+1}^{r-1} \boldsymbol{P}_{\tilde{x}_{k+1 \mid k+1}^{r-1}+\tilde{x}_{j, k+1 \mid k+1}^{r-1}}
$$

\section{Remark 3.1}

If the initial conditions of the estimator are far from the actual initial conditions of the system, and since the projection on the innovation space is not orthogonal owing to the linearization and the different approximations made during the implementation of this phase, the filter phase of the lemma 3.1 (the third and fourth steps) have to be applied repetitively for a few number of iteration using the same output measurement at the same sampling instant [14].

\subsection{The Dynamics of the Decomposed Nonlinear Update Estimator}

Let us assume that a subset of $\mathrm{z}$ constraints of the imposed $q$ constraints given by (4) are not satisfied. To insure the satisfaction of the constrained estimation problem, the violated subset of constraints has to be saturated to the corresponding upper or lower bounds imposed in (4). Therefore, we impose the following set of $z$ equality constraints to be satisfied:

$$
\theta_{k+1}=m_{k+1}\left(x_{k+1}\right)
$$

where $\boldsymbol{m}_{k+1}\left(\boldsymbol{x}_{k+1}\right): \boldsymbol{R}^{n} \rightarrow \boldsymbol{R}^{z}$ is a vector function containing the $z$ violated constraints from (4). The vector $\theta_{k+1} \in \boldsymbol{R}^{z}$ contains the corresponding upper or lower bounds which have been violated in (4).

Equation (21) will be treated as a new received set of measurements to be used for the nonlinear update of the estimator.

By decomposing (21) into $p$ sub-vectors, we get:

$$
\theta_{\alpha, k+1}=\boldsymbol{m}_{\alpha, k+1}\left(x_{k+1}\right)
$$

where $\boldsymbol{m}_{\alpha, k+1}\left(\boldsymbol{x}_{k+1}\right): \boldsymbol{R}^{n} \rightarrow \boldsymbol{R}^{z_{\alpha}}, \theta_{\alpha, k+1} \in \boldsymbol{R}^{z_{\alpha}}$ are the $\alpha^{\text {th }}$ sub-vector of (21), $z=\sum_{\alpha=1}^{p} z_{\alpha}$ and $\theta_{k+1}^{T}=\left[\theta_{1, k+1}^{T}, \theta_{2, k+1}^{T}, \ldots, \theta_{\alpha, k+1}^{T} \ldots, \theta_{p, k+1}^{T}\right]$.

Therefore, and due to the nonlinearity of the system, the nonlinear update estimator for the $i^{\text {th }}$ subsystem 
is approximated by:

$$
\hat{\boldsymbol{x}}_{\boldsymbol{i}, \boldsymbol{k}+1 \mid \boldsymbol{k}+1}=\boldsymbol{E}\left\{\boldsymbol{x}_{\boldsymbol{i}, \boldsymbol{k}+1} \mid \boldsymbol{Y}^{\boldsymbol{k}}, \boldsymbol{y}_{\boldsymbol{k}+1}, \theta_{1, \boldsymbol{k}+1}, \theta_{2, \boldsymbol{k}+1}, \ldots, \theta_{p, \boldsymbol{k}+1}\right\}
$$

Let us denote the estimator ${ }^{\hat{\boldsymbol{x}}_{\boldsymbol{i}, \boldsymbol{k}+1 \mid \boldsymbol{k}+1}}$ and the estimation error $\tilde{\boldsymbol{x}}_{\boldsymbol{i}, \boldsymbol{k}+1 \mid \boldsymbol{k}+1}$ resulting from the IDUEKF by $\hat{\boldsymbol{x}}_{\boldsymbol{i}, \boldsymbol{k}+1 \mid \boldsymbol{k}+1}^{\prime}$ and $\tilde{\boldsymbol{x}}_{\boldsymbol{i}, \boldsymbol{k}+1 \mid \boldsymbol{k}+1}^{\prime}$, respectively. Although $\tilde{\boldsymbol{x}}_{\boldsymbol{i}, k+1 \mid k+1}^{\prime}$ depends on the parameter vector $\boldsymbol{c}_{\boldsymbol{k}+1}$ as well as the measurement noise $v_{k+1}$, an approximation will be made in which it will be assumed that $\tilde{\boldsymbol{x}}_{\boldsymbol{i}, \boldsymbol{k}+1 \mid \boldsymbol{k}+1}^{\prime}$ is independent on $\tilde{\boldsymbol{c}}_{\boldsymbol{k}+1}$ and $\boldsymbol{v}_{\boldsymbol{k}+1}$ while linearizing $\boldsymbol{m}_{\alpha, \boldsymbol{k}+1}\left(\boldsymbol{x}_{\boldsymbol{k}+1}\right)$ around $\hat{\boldsymbol{x}}_{\boldsymbol{k}+1 \mid \boldsymbol{k}+1}^{\prime} ; \overline{\boldsymbol{c}}$. Moreover, during the implementation of the algorithm, singular matrices can be encountered due to the approximation made by neglecting the higher order terms in the linearization process, and the assumption that $\tilde{\boldsymbol{x}}_{\boldsymbol{i}, \boldsymbol{k}+1 \boldsymbol{k}+1}^{\prime}$ is independent of $\boldsymbol{c}_{\boldsymbol{k}+1}$ and $\boldsymbol{v}_{\boldsymbol{k}+1}$. To avoid such a singularity, a zero mean Gaussian random variable $\boldsymbol{s}_{\alpha, \boldsymbol{k}+1}$ with covariance matrix $\boldsymbol{S}_{\alpha, k+1}$ will be added to the imposed constraint equation (21). Such a covariance matrix will be given small numbers.

\section{Lemma 3.2}

For sub-vector of the violated constraints $\theta_{\alpha, k+1}$ where $\alpha \in\{1,2, \ldots . p\}$ :

1) The decomposed nonlinear update estimators are given by:

$$
\begin{aligned}
& \hat{\boldsymbol{x}}_{i, k+1 \mid k+1}^{\prime \alpha}=\hat{\boldsymbol{x}}_{i, k+1 \mid k+1}^{\prime \alpha-1}+\boldsymbol{K}_{2_{i, k+1}^{\alpha}}^{\alpha} \tilde{\boldsymbol{\theta}}_{\alpha, k+1 \mid k+1}^{\alpha-1} \\
& \hat{\boldsymbol{x}}_{\boldsymbol{i}, \boldsymbol{k}+1 \mid \boldsymbol{k}+1}^{\prime 0}=\hat{\boldsymbol{x}}_{\boldsymbol{i}, \boldsymbol{k}+1 \mid \boldsymbol{k}+1}^{\prime} \text { and } \hat{\boldsymbol{x}}_{\boldsymbol{i}, \boldsymbol{k}+1 \mid \boldsymbol{k}+1}=\hat{\boldsymbol{x}}_{\boldsymbol{i}, \boldsymbol{k}+1 \mid \boldsymbol{k}+1}^{p} \\
& \tilde{\theta}_{\alpha, k+1 \mid k+1}^{\alpha-1}=\theta_{\alpha, k+1}-\boldsymbol{m}_{\alpha, k+1}\left(\hat{\boldsymbol{x}}_{\boldsymbol{k}+1 \mid \boldsymbol{k}+1}^{\alpha-1}\right) \\
& \boldsymbol{K}_{2_{i, k+1}}^{\alpha} \cong \boldsymbol{P}_{\tilde{x}_{i, k+1 \mid k+1}^{\prime \alpha-1} \tilde{x}_{k+1 \mid k+1}^{\prime r-1}}^{\prime \alpha-1} \hat{\boldsymbol{M}}_{\alpha, k+1}^{\alpha-1^{T}} \times\left[\hat{\boldsymbol{M}}_{\alpha, k+1}^{\alpha-1} \boldsymbol{P}_{\tilde{x}_{k+1 k+1}^{\prime \alpha-1} \tilde{x}_{k+1 \mid k+1}^{\prime \alpha-1}}^{\prime \alpha-1} \hat{M}_{\alpha, k+1}^{\alpha-1^{T}}+\boldsymbol{S}_{\alpha, k+1}\right]^{-1} \\
& \hat{\boldsymbol{M}}_{\alpha, k+1}^{\alpha-1}=\left[\left.\frac{\partial \boldsymbol{m}_{\alpha, k+1}^{T}}{\partial \boldsymbol{x}_{k+1}}\right|_{\hat{x}_{k+1}=\hat{x}_{k+1 \mid k+1}^{\prime \alpha-1}}\right]^{T}
\end{aligned}
$$

where $\boldsymbol{P}_{\tilde{x}_{k+1 \mid k+1}^{\prime 0} \tilde{x}_{k+1 \mid k+1}^{\prime 0}}^{\prime 0}=\boldsymbol{P}_{\tilde{x}_{k+1 \mid k+1} \tilde{x}_{k+1 \mid k+1}}$ is the covariance matrix at the end of the iterations of the IDUEKF. 2-a) The covariance matrices of the filtered error of the $i^{\text {th }}$ subsystems (for $i \in\{1,2, . ., N\}$ ) are given by:

$$
\boldsymbol{P}_{\tilde{x}_{i, k+1 \mid k+1}^{\prime \alpha}}^{\prime \alpha} \tilde{x}_{i, k+1 \mid k+1}^{\prime \alpha}=P_{\tilde{x}_{i, k+1 \mid k+1}^{\prime \alpha-1} \tilde{x}_{i, k+1 \mid k+1}^{\prime \alpha-1}}^{\prime \alpha-1}-\boldsymbol{K}_{2_{i, k+1}}^{\alpha} \hat{M}_{\alpha_{k+1}}^{\alpha-1} \boldsymbol{P}_{\tilde{x}_{k+1 \mid k+1}^{\prime \alpha-1} \tilde{x}_{i, k+1 \mid k+1}^{\prime \alpha-1}}^{\prime \alpha-1}
$$

2-b) The cross covariance matrices of the filtered error of the $i j^{\text {th }}$ subsystems (for $j \in\{i+1,2, . ., N\}$ are given by:

$$
P_{\tilde{x}_{i, k+1 \mid k+1}^{\prime \alpha} \tilde{x}_{j, k+1 \mid k+1}^{\prime \alpha}}^{\prime \alpha}=P_{\tilde{x}_{i, k+1 \mid k+1}^{\prime \alpha-1} \tilde{x}_{j, k+1 \mid k+1}^{\prime \alpha-1}}^{\prime \alpha-1}-\boldsymbol{K}_{2_{i, k+1}}^{\alpha} \hat{M}_{\alpha_{k+1}}^{\alpha-1} \boldsymbol{P}_{\tilde{x}_{k+1 \mid k+1}^{\prime \alpha-1} \tilde{x}_{j, k+1 \mid k+1}^{\prime \alpha-1}}^{\prime \alpha-1}
$$

\section{Simulation Results}

\subsection{Phase Portrait of the Proposed System}


The system is simulated in order to show the impact of the uncertainties of the parameters and the application of the constraints on the performance of the proposed uncertain constrained hyperchaotic system. The implemented adaptive formula of $\alpha$ is as given by (3). The parameters $a, b, c, d$ and $r$ are assumed to be uncertain with the following statistics: $a \sim N(35,12.25), b \sim N(3,0.09)$, $c \sim N(12,1.44), d \sim N(7,0.49)$, and $r \sim N(0.5,0.0025)$. In our simulation, the sampling period $\Delta T$ is taken to be equal to $0.0001 \mathrm{sec}$. The sets of constraints are chosen to take the form:

For the unified chaotic subsystem,

$$
\begin{aligned}
& g_{1 k+1}=x_{u 1 k+1}^{2}-x_{u 2 k+1}^{2}+x_{u 3 k+1}^{2} \\
& 5 \times 10^{3} \leq k+1<7.5 \times 10^{5} \quad g_{1 k+1} \leq 1100+0.0003(k+1-50) \\
& 7.5 \times 10^{5} \leq k+1<3.5 \times 10^{6} \quad g_{1 k+1} \leq 1400+200 \cos \left(\left(k+1-2.5 \times 10^{6}\right) 5 \times 10^{-6}\right) \\
& 3.5 \times 10^{6} \leq k+1<6 \times 10^{6} \quad g_{1 k+1} \leq 1800+200 \sin \left(\left(k+1-5 \times 10^{6}\right) 7 \times 10^{-6}\right) \\
& \text { for other values of } k \text { the system is free. }
\end{aligned}
$$

For the hyperchaotic Chen subsystem:

$$
\begin{array}{lr}
5 \times 10^{3} \leq k+1<4 \times 10^{6} & g_{2 k+1}=x_{h 1 k+1} \leq 15 \\
4 \times 10^{6} \leq k+1<7.5 \times 10^{6} & g_{2 k+1}=x_{h 1 k+1} \leq 13 \\
7.5 \times 10^{6} \leq k+1<8.5 \times 10^{6} & g_{2 k+1}=x_{h 1 k+1} \leq 12 \\
\text { for other values of } k \text { the system is free. }
\end{array}
$$

In Fig. 1, different views of the attractor's phase diagram of the proposed system are shown. It can be observed that the constrained coupled hyperchaotic system maintains its chaotic behavior.

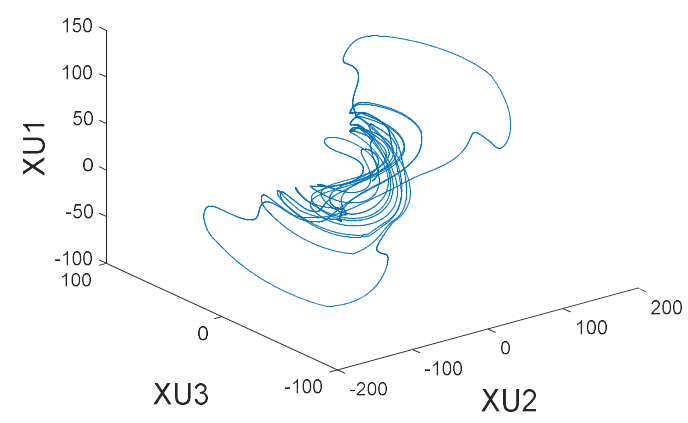

(a)

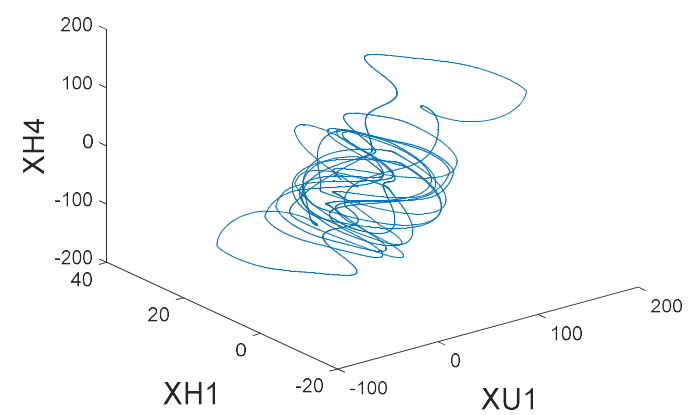

(c)

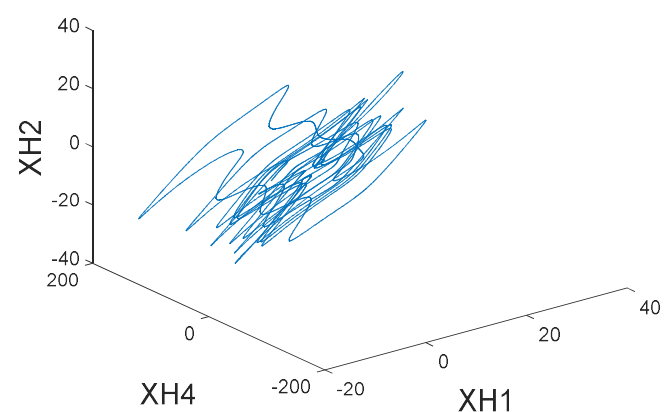

(b)

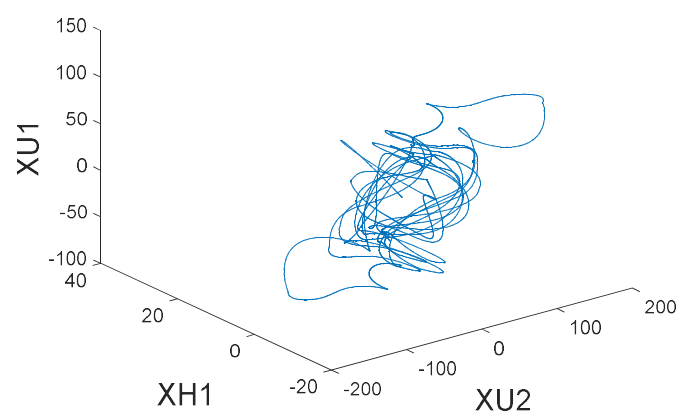

(d)

Fig. 1. Different views of the phase diagram of the proposed constrained coupled hyperchaotic system. 


\subsection{Case Study}

A simulated case study is presented in which two coloured images are assumed to be transmitted from the transmitter to the receiver end using the proposed chaos-based secure communications scheme.

Before this process, it is necessary to re-dimension the images to become a binary sequence and re-scale the binary sequences to become pulses with width $5 \Delta T$. Hence the pulse is:

$$
S(k)=\left\{\begin{array}{lll}
+0.5 & \text { if the bit is } 1 & k \in\left[k_{o}, k_{o}+5 \Delta T\right. \\
-0.5 & \text { if the bit is } 0 & k \in\left[k_{o}, k_{o}+5 \Delta T\right]
\end{array}\right.
$$

The generated data pulses are encrypted using the following formula:

$$
S T(k)=0.1 S(k)\left|x_{h 1}(k)+30\right|
$$

where $S T(k)$ is the encrypted data signal. The modulated output $y_{h 2 k+1}^{m}$ is such that

$$
y_{h 2 k+1}^{m}(k)=0.1 x_{h 2 k+1}(k)+S T(k)
$$

The modulation process starts at $t=5 \mathrm{sec}$. The applied encryption rule of the data signal is agreed upon between the transmitter and the receiver. In our case, the following encryption rules are used:

$$
\begin{aligned}
& y_{u 1 k+1}^{e}=y_{u 1 k+1}+2 y_{h 2 k+1}-1.5 y_{h 1 k+1}+v_{1 k+1} \\
& y_{u 2 k+1}^{e}=y_{u 1 k+1}^{e}+y_{u 2 k+1}-2 y_{h 1 k+1}+v_{2 k+1} \\
& y_{h 1 k+1}^{e}=2.5 y_{h 1 k+1}-y_{u 1 k+1}-2 y_{h 2 k+1}+0.1 \sin \left(\left|0.4 x_{u 3 k}\right|\right)+v_{3 k+1} \\
& y_{h 2 k+1}^{e}=y_{h 1 k+1}^{e}+3 y_{h 2 k+1}+y_{u 1 k+1}+v_{4 k+1}
\end{aligned}
$$

where $y_{u 1 k+1}^{e}$ and $y_{u 2 k+1}^{e}$ are the encrypted outputs of the unified chaotic oscillator, $y_{h 1 k+1}^{e}$ and $y_{h 2 k+1}^{e}$ are the encrypted outputs of the Chen hyperchaotic oscillator and $\boldsymbol{v}_{\boldsymbol{k}} \in \boldsymbol{R}^{4}$ is a zero mean white Gaussian output noise vector with covariance matrix $\boldsymbol{Q}=\boldsymbol{E}\left\{\boldsymbol{v} \boldsymbol{v}^{T}\right\}$.

At the receiving end the decrypted outputs $y_{u 1 k+1}^{d}, y_{u 2 k+1}^{d}, y_{h 1 k+1}^{d}$ are used to estimate the state vectors $\hat{\boldsymbol{x}}_{\boldsymbol{u}+1 \mid k+1}, \hat{\boldsymbol{x}}_{\boldsymbol{k} k+1 k+1}$ and hence, the output $\hat{y}_{h 2 k+1 k+1}=0.1 \hat{x}_{h 2 k+1 k+1}$. Then by direct subtraction between the decrypted output $y_{h 2 k+1}^{d m}$ and $\hat{y}_{h 2 k+1 k+1}$, we retrieve $S \hat{T}(k)$ and then $\hat{S}(k)$ using the following decryption rule:

$$
\hat{S}(k)=S \hat{T}(k) /\left(0.1\left|\hat{x}_{h 1}(k)+30\right|\right)
$$

The signal $\hat{S}(k)$ is applied to the filter and the output is used to retrieve the images by re-dimensioning the recovered binary sequences back to become $n \times m \times l$ matrix where $l=24$ is a sequence of 24 bits to identify the color of each pixel, $n$ and $m$ are the numbers of rows and columns of the image matrix, respectively. The quality of the communication scheme is measured using the BER defined by:

$$
\text { BER }=\frac{\text { No.of miss }- \text { matched bits }}{\text { nxmxl }} 100 \%
$$

The proposed system is simulated using the sampling period $\Delta T=0.0001 \mathrm{~s}$, the same statistics of the

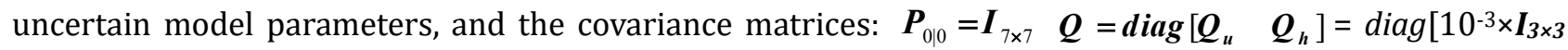
$\left.1^{-4 \times \mathbf{I}_{4 \times 4}}\right]$, and $\boldsymbol{R}=0.025 \times \mathbf{I}_{4 \times 4}$. The initial condition of the state vector $\boldsymbol{x}_{o}=\boldsymbol{x}(0)=\left[\begin{array}{lllllll}1 & 1 & 1 & 1 & 1 & 1 & 1\end{array}\right]$, while 
that of the estimator is $\hat{\boldsymbol{x}}_{\boldsymbol{o} \boldsymbol{o}}=\left[\begin{array}{lllllll}0 & 0 & 0 & 0 & 0 & 0 & 0\end{array}\right]$.

The actual and the retrieved images are presented in Fig. 2 and 3. The BERs are $0.022515 \%$ and $0.019097 \%$, respectively. The results indicate that the quality of the retrieved images is very satisfactory and that the IDUCEKF is capable of reconstructing the states of the uncertain constrained coupled hyperchaotic system very efficiently. The quality of the communications scheme can also be quantitatively assessed by evaluating the correlation factor between the transmitted and received images which was found to be equal 0.999 for both images. More importantly, the correlation factors between the transmitted/received images and the channel data of the encrypted carrier $y_{h 2}^{e}(k+1)$ were evaluated and found to be 0.499996 and 0.498410 for images 1 and 2, respectively. These numbers were found to be 0.07022 and 0.07337 if the encrypted carrier $y_{h 2}^{e}(k+1)$ is changed to take the form:

$$
y_{h 2}^{e}(k+1)=y_{h 2}(k+1)-15 y_{u 1}(k+1)+5 y_{h 1}(k+1)+v_{4}(k+1)
$$

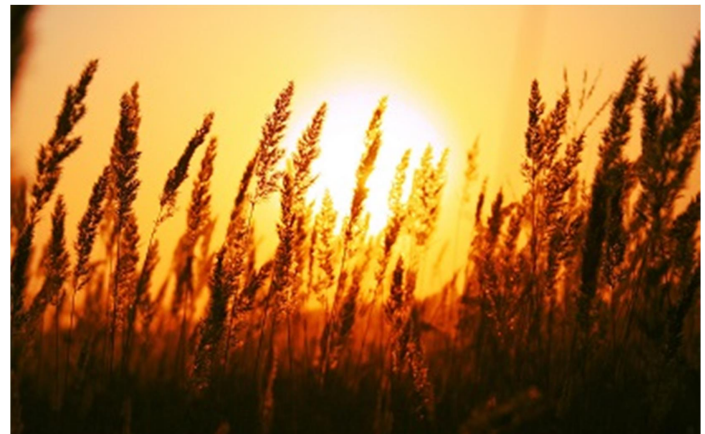

(a)

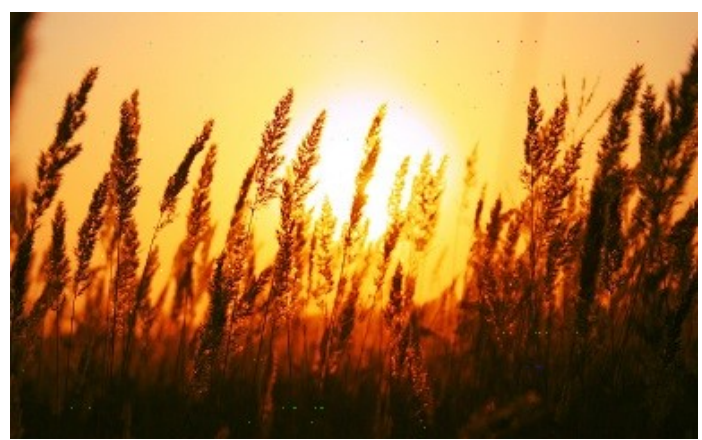

(b)

Fig. 2. Image 1. (a) Actual image. (b) Retrieved image.

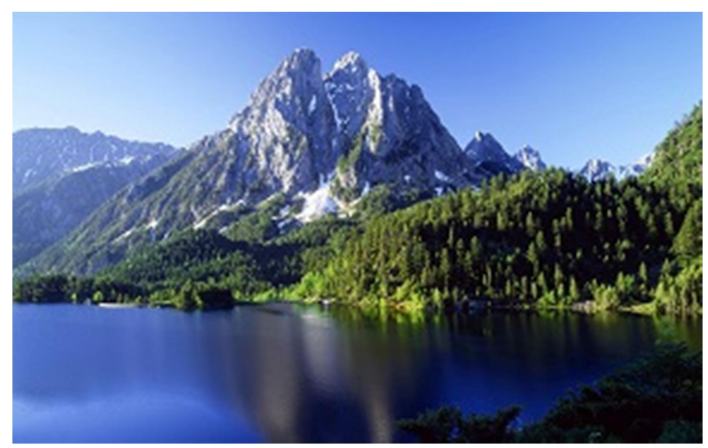

(a)

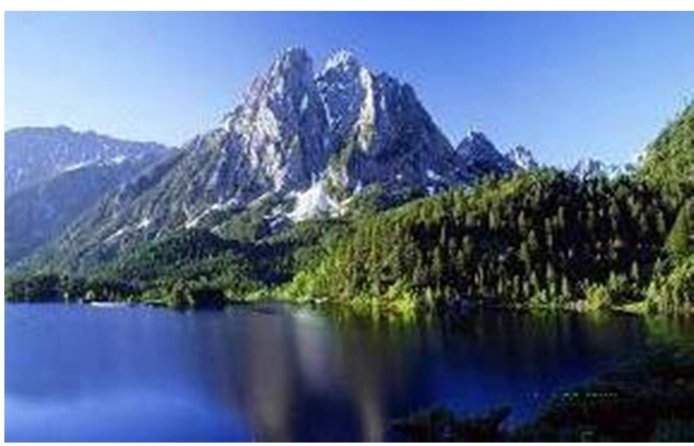

(b)

Fig. 3. Image 2. (a) Actual image. (b) Retrieved image.

\section{Conclusions}

In this paper, the unified chaotic and the hyperchaotic Chen systems are coupled, constrained and used as a new hyperchaotic system in a secure communication scheme. The outputs of the system are encrypted and transmitted to the receiving end through a noisy public communication channel. At the receiving end, the IDUCEKF is used to reconstruct the constrained hyperchaotic signals, and hence retrieve the transmitted data. The proposed secure communication scheme is applied to transmit discrete images, and the quality of the retrieved images, measured by the BER and the associated correlation coefficients, showed its applicability in reality and the effectiveness of the IDUCEKF in estimating the states of the uncertain constrained hyperchaotic system. 


\section{Acknowledgment}

This work was supported by the Kuwait University under research Grant no. EE 02/14.

\section{References}

[1] Chang, W. D., Shih, S. P., \& Chen, C. Y. (2015). Chaotic secure communication systems with an adaptive state observer. J. Control Science and Engineering, Article 15.

[2] Fallahi, K., Raoufi, R., \& Khoshhin, H. (2008). An application of Chen system for secure chaotic communication based on extended Kalman filter and multi-shift cipher algorithm. Communication in Nonlinear Science and Numerical Simulation,13, 763 - 781.

[3] Wang, B., Zhong, S. M., \& Dong, X. C. (2016). On the novel chaotic secure communication scheme design. Communication in Nonlinear Science and Numerical Simulation, 39, 108 - 117.

[4] Martínez-Guerra, R., García, J. M., \& Prieto, S. M. (2016). Secure communications via synchronization of Liouvillian chaotic systems. Journal of the Franklin Institute, 353(17), 4384 - 4399.

[5] Smaoui, N., Karouma, A., \& Zribi, M. (2011). Secure communication based on the synchronization of the hyperchaotic Chen and the unified chaotic systems. Communication in Nonlinear Science and Numerical Simulation, 16, 3279 - 3293.

[6] Wang, B., \& Dong, X. (2015). Secure communication based on a hyperchaotic system with disturbances. Math. Problems in Engineering, 616137.

[7] Ruan, H., Zhai, T., \& Yaz, E. E. (2003). A chaotic secure chaotic communication scheme with extended Kalman filter based parameter estimation. Proceedings of the IEEE Conf. on Control Applications, 1 , $404-408$.

[8] Zhu, Z., \& Leung, H. (2001). Adaptive blind equalization for chaotic communication systems using extended Kalman filter. IEEE Trans. Circuits and Syst, I, (48), 979 - 987.

[9] Leung, H., Zhu, Z., \& Ding, Z. (2000). An aperiodic phenomenon of the extended Kalman filter in filtering noisy chaotic signals. IEEE Trans. Signal Processing, 48(6), 1807 - 1810.

[10] Calitoiu, D., Oommen, B. J., \& Nussbaum, D. (2007). Desynchronizing a chaotic pattern recognition neural network to model inaccurate perception. IEEE Trans. Syst. Man and Cyber, Part B, 37(3), 692 704.

[11] Hassan, M. F. (2014). A new approach for secure communication using constrained hyperchaotic systems. Applied Mathematics and Computation, 246, 711 - 730.

[12] Short, K. M. (1996). Unmasking a modulated chaotic communication scheme. Int. J. of Bifurcat Chaos, 6, $367-375$.

[13] Perez, G., \& Cerdeira, H. A. (1995). Extracting messages masked by chaos. Phys Rev. Lett., 74, 1970 1973.

[14] Hassan, M. F., Alrifai, M. T., Soliman, H. M., \& Kourah, M. A. (2016). Observer-based controller for constrained uncertain stochastic nonlinear discrete-time systems. Int. J. of Robust Nonlinear Control, 26(10), $2090-2115$.

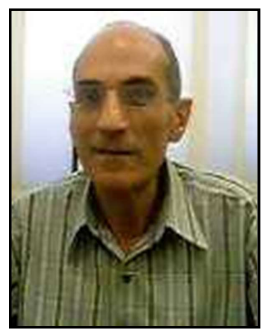

Mohamed F. Hassan was born in Cairo, Egypt on June 21, 1947. He received the B.Sc. and M.Sc. degrees in electrical engineering from Cairo University, Faculty of Engineering, Cairo, Egypt in 1970 and 1973 respectively, and D.Sc. (Doctor d'Etat) in systems science and automatic control from Paul Sabatier University, Toulouse, France, 1978.

He has been a faculty member in Electronics and Communication Department, Faculty of Engineering, Cairo University since 1978 where he has been a professor since 1988. 
Currently, he is on leave from Cairo University and has joined Kuwait University. He is an author and co-author of more than 165 technical papers in most of the international journals and conferences, a book, and 7 book chapters. His current research interests are in the areas of large scale systems, optimization theory, stochastic control theory, estimation theory, and nonlinear systems.

Prof. Hassan is a member of the Egyptian Engineering Society and the Egyptian Society for Applied Operation research. He was the recipient of the 1981 Science State Prize for outstanding research in Engineering and holds the State Medal of Science and Arts (first class), Egypt. He was nominated to receive the distinct "American Medal of Honor" from the American Biographical Institute, June 26, 2002. He has been listed in the 1983 edition of Mathematics: Who's who, in the 2007 Academic Keys Who's Who in Engineering Higher Education, and also in the 2007 Who's Who in Science and Engineering. 